\section{Nefrectomía radical: incidencia de morbilidad y mortalidad en un hospital de tercer nivel}

Calvo-Vázquez I, Santander-Flores SA, González-Villegas P, Bravo-López GM, Yáñez-Sosa AL, Reyna-Blanco I

Resumen

ANTECEDENTES: la tasa de complicaciones por nefrectomía radical es de $20 \%$ y de ésta, $5 \%$ se consideran graves.

OBJETIVO: determinar la incidencia de morbilidad y mortalidad perioperatoria por nefrectomía radical en pacientes con cáncer renal.

MATERIALES Y MÉTODOS: estudio observacional, ambispectivo, en el que se revisaron los expedientes clínicos de pacientes con cáncer renal y nefrectomía radical operados en el Hospital Central Norte de PEMEX entre el 1 de julio de 2009 y el 30 de junio de 2015. Se evaluaron las complicaciones trans y posoperatorias y se clasificaron según su severidad.

RESULTADOS: se registraron 89 expedientes, de los que 71 cumplieron con los criterios de inclusión. Se registraron complicaciones transoperatorias en $25.4 \%$ de los casos, principalmente el desgarro de vasos de neoformación y adyacentes $(8.5 \%)$, y $33.8 \%$ postoperatorias. La mayoría de los pacientes se clasificaron en estadio Gl y GIII; solo $11.3 \%$ en GIV y $4.2 \%$ de mortalidad (GV).

CONCLUSIONES: la mayor parte de las complicaciones fueron de grado bajo (Gl a GIII). El sistema estandarizado para clasificación por grado de severidad representa una herramienta útil para evaluar los procedimientos quirúrgicos del cáncer renal.

PALABRAS CLAVE: cáncer renal, nefrectomía radical, complicaciones posoperatorias.

Rev Mex Urol. 2017 Nov-Dec;77(6):446-452.

\section{Radical nephrectomy: Incidence of morbidity and mortality in a tertiary hospital}

Calvo-Vázquez I, Santander-Flores SA, González-Villegas P, Bravo-López GM, Yáñez-Sosa AL, Reyna-Blanco I

\section{Abstract}

BACKGROUND: The complication rate for radical nephrectomy is $20 \%$, and $5 \%$ of the complications are severe.
Servicio de Cirugía, Hospital Central Norte de PEMEX, Ciudad de México.

Recibido: enero 2017

Aceptado: septiembre 2017

Correspondencia

Iván Calvo-Vázquez

dr.ivancv_unam@hotmail.com

Este artículo debe citarse como

Calvo-Vázquez I, Santander-Flores SA, GonzálezVillegas P, Bravo-López GM, Yáñez-Sosa AL, ReynaBlanco I. Nefrectomía radical: incidencia de morbilidad y mortalidad en un hospital de tercer nivel. Rev Mex Urol. 2017 nov-dic;77(6):446-452.

DOI: https://doi.org/10.24245/revmexurol.v77i6.1177 
AlM: To determine the incidence of perioperative morbidity and mortality of radical nephrectomy in patients with kidney cancer.

MATERIALS AND METHODS: An observational, ambispective study was conducted, in which the case records were reviewed of all patients diagnosed with kidney cancer that underwent radical nephrectomy at the Hospital Central Norte de PEMEX within the time frame of July 1 , 2009 and June 30, 2015. The intraoperative and postoperative complications were evaluated and classified according to their severity.

RESULTS: Of the 89 case records reviewed, 71 met the inclusion criteria. A total of $25.4 \%$ of the cases presented with intraoperative complications, and the most frequent was the tearing of newly formed vessels and those adjacent to them (8.5\%). There were postoperative complications in $33.8 \%$ of the cases. Most of the complications were grade I and grade III. Only $11.3 \%$ were classified as grade IV, and $4.2 \%$ as grade $\mathrm{V}$.

CONCLUSIONS: The majority of complications were low-grade (grade I to grade III). Assigning a severity grade to complications through a standardized classification system is useful in the evaluation of surgical procedures for treating kidney cancer.

KEYWORDS: Kidney cancer; Radical nephrectomy; Postoperative complications
Servicio de Cirugía, Hospital Central Norte de PEMEX, Ciudad de México.

Correspondence Iván Calvo-Vázquez dr.ivancv_unam@hotmail.com

\section{ANTECEDENTES}

Los tumores renales ocupan el decimocuarto lugar en incidencia y el decimosexto en mortalidad a nivel mundial. Según el programa Surveillance, Epidemiology, and End Results (SEER), la incidencia es de 15.1/100,000, con edad media de manifestación a los 64 años. De acuerdo con el INEGI, México ocupa el duodécimo lugar en mortalidad, con un índice de 0.52 , es decir, de todos los cánceres que se diagnostican al año, 2.5\% son de cáncer renal y de éste, $50 \%$ son mortales. ${ }^{1}$

En los últimos años el diagnóstico se ha establecido de manera fortuita, mediante imágenes evaluadas por alteraciones abdominales. ${ }^{2}$ Desde el punto de vista histológico, $85 \%$ de las neoplasias corresponden a carcinoma de células renales, sobre todo el carcinoma de células claras (80\%), seguido del papilar (10-15\%) y de células cromófobas ( $5 \%)$. Otros tumores de células renales pertenecen a carcinoma de túbulos colectores y medulares..$^{3,4}$ La principal manifestación consiste en la triada de dolor abdominal, hematuria y masa palpable en el flanco; sin embargo, estudios recientes reportan que solo $10 \%$ de los pacientes expresan estos síntomas. ${ }^{3}$ Actualmente, el síntoma más frecuente es la hematuria (50\% de los casos)..$^{5} \mathrm{Al}$ momento del diagnóstico 20 a $25 \%$ de los pacientes tiene enfermedad metastásica, principalmente a los pulmones, hígado, huesos y sistema nervioso central. Los pacientes con enfermedad local tienen $30 \%$ de probabilidad de recidiva después de la nefrectomía radical. ${ }^{6}$ La mortalidad específica de la enfermedad es cercana a $40 \%$, la supervivencia global a 5 años de $45-65 \%$ y la metástasis disminuye, incluso, a $20 \%{ }^{7}$ 
La cirugía sigue siendo el tratamiento de primera línea en sujetos con neoplasias renales. En 1969, Robson estableció la nefrectomía radical como el tratamiento de referencia para el cáncer renal localizado. ${ }^{8}$ El concepto de nefrectomía radical tiene los principios básicos de la ligadura de arterias y venas renales, extirpación del riñón (incluida la fascia de Gerota) y de la glándula suprarrenal ipsilateral y linfadenectomía regional completa, desde la crura del diafragma hasta la bifurcación de la aorta. La tasa de complicaciones se estima en $20 \%$, del que $5 \%$ se consideran graves y $15 \%$ leves. $^{6}$

El objetivo de este estudio es estimar la morbilidad y mortalidad de pacientes con cáncer renal con nefrectomía radical, y evaluar la supervivencia específica de la enfermedad.

\section{MATERIALES Y MÉTODOS}

Estudio observacional y ambispectivo en el que se analizaron los expedientes clínicos de pacientes con diagnóstico de cáncer renal y nefrectomía radical operados entre el 1 de julio de 2009 y el 30 de junio de 2015 en el Hospital Central Norte de PEMEX.

Se determinó la incidencia de complicaciones asociadas con la nefrectomía radical, describiéndolas y estableciendo factores adicionales como: requerimiento de trasfusión sanguínea, ingreso a la unidad de terapia intensiva y tasa de mortalidad. Se analizaron las complicaciones perioperatorias, es decir, las que ocurrieron en el primer mes del posoperatorio. Para el análisis estadístico se utilizó el programa SPSS.

Criterios de inclusión: pacientes con diagnóstico de cáncer renal intervenidos para nefrectomía radical entre el 1 de julio de 2009 y el 30 de junio de 2015. Criterios de exclusión: sujetos que no recibieron tratamiento quirúrgico y quienes tuvieron expediente clínico incompleto.
Variables analizadas: sexo, edad, sitio de la neoplasia, tiempo quirúrgico, sangrado transoperatorio, requerimiento de trasfusión sanguínea, complicaciones transoperatorias, mortalidad, ingreso a la unidad de cuidados intensivos (UCI) y días de estancia intrahospitalaria; además, se obtuvo el reporte histopatológico y estadio de la neoplasia. Con las notas de evolución de los días subsecuentes al procedimiento se determinaron las complicaciones posoperatorias. Como dato final, se tomaron en cuenta las valoraciones en consulta externa para estimar las complicaciones ocurridas en los primeros 30 días del posoperatorio.

Las complicaciones se clasificaron según el sistema de Clavien-Dindo, que considera 5 grados más dos subgrupos de los grados III y IV. Las complicaciones de grado I producen una desviación del curso normal del postoperatorio, pero no requieren intervención quirúrgica, endoscópica o radiológica, solo la prescripción de medicamentos básicos. Las de grado II requieren tratamiento farmacológico, trasfusión o nutrición parenteral total. Las complicaciones grado III suponen intervención quirúrgica, endoscópica o radiológica. Las complicaciones que requieren tratamiento intensivo corresponden al grado IV y las que ocasionan la muerte al grado $\mathrm{V}$.

\section{RESULTADOS}

Se revisaron 89 expedientes, de los que 71 cumplieron con los criterios de inclusión. Se registraron 40 (56.3\%) hombres y $31(43.7 \%)$ mujeres. El lado izquierdo fue el más afectado $(n=44[62 \%])$, mientras que el derecho solo afectó a 27 (38\%) casos. El promedio de edad fue de 65.6 años ( $D E \pm 13.352$, límites de 34-87 años) Figura 1.

El promedio de tiempo quirúrgico fue de 153.4 minutos (límites de 75 y 330 min) y de estancia 


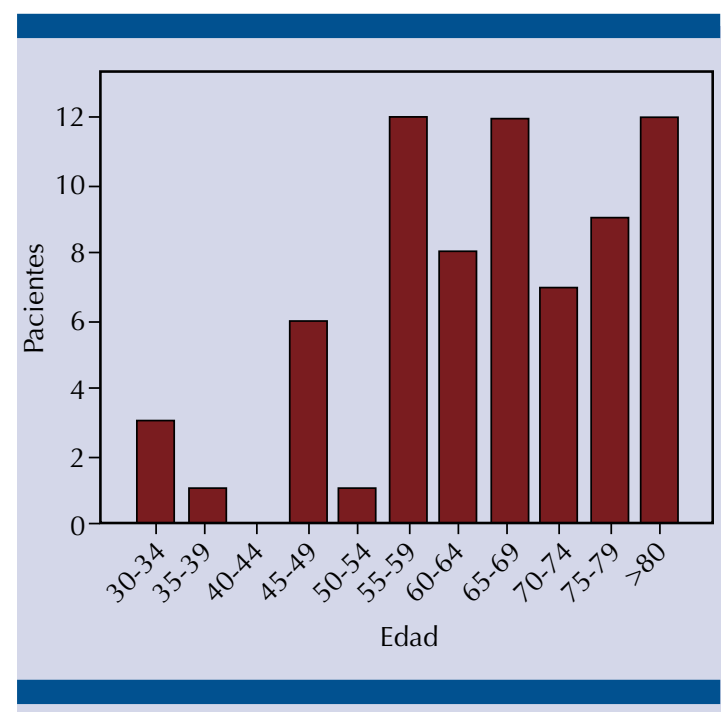

Figura 1. Distribución por grupos de edad.

hospitalaria de 9.1 días (5 a 18 días). El sangrado transoperatorio estimado fue de $1116.4 \mathrm{cc}(100 \mathrm{a}$ 6000 cC; DE \pm 1436.109$) ; 18$ pacientes $(25.4 \%)$ requirieron trasfusión y el resto $(74.6 \%)$ no la ameritó durante el posoperatorio.

Las complicaciones transoperatorias ocurrieron en $25.4 \%$ de los casos, principalmente: desgarro de los vasos de neoformación y adyacentes (8.5\%), laceración del bazo (5.6\%), desgarro de la vena cava $(4.2 \%)$ y laceración diafragmática (2.8\%). En menor frecuencia se encontró: lesión de la arteria suprarrenal, desgarro de pleura ipsilateral y tumor con absceso en $1.4 \%$, respectivamente (Figura 2).

Durante el posoperatorio se registraron 33.8\% de complicaciones, principalmente: hemorragia e hipotensión (40\%), insuficiencia respiratoria aguda $(15.5 \%)$, insuficiencia renal aguda $(11.1 \%)$, neumotórax $(8.9 \%)$, insuficiencia cardiaca $(6.7 \%)$, neumonía $(6.7 \%)$; dehiscencia de la herida, sepsis abdominal, atelectasia, retención aguda de orina, hematoma e infección de herida quirúrgica $(2.2 \%)$, incluso se reportaron pacien-

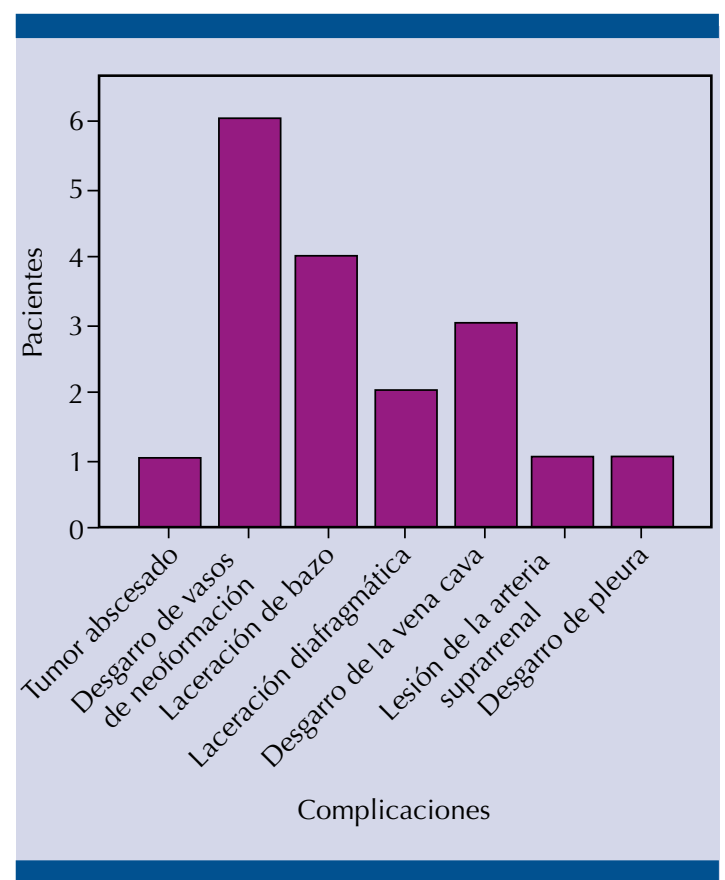

Figura 2. Complicaciones transoperatorias.

tes con más de una complicación (Cuadro 1). Según su gravedad, la mayor parte de las complicaciones trans y posoperatorias se clasificaron como Gl y GIII. El $11.3 \%$ correspondió a GIV y $4.2 \%$ a GV.

Ocho pacientes ameritaron ingresar a la unidad de cuidados intensivos $(11.3 \%$ ) y el resto permaneció hospitalizado en piso (88.7\%). La tasa de mortalidad fue de $4.2 \%(n=3)$ y de supervivencia de $95.8 \%(n=68)$.

Los estudios histopatológicos reportaron 62 $(87.3 \%)$ pacientes con carcinoma de células claras, $6(8.5 \%)$ con carcinoma renal de los conductos colectores y 3 (4.2\%) con carcinoma de células cromófobas (Cuadro 2). El estadio con mayor frecuencia detectado fue T1 a y T2a (21.1\%, respectivamente), seguido de T3a (18.3\%), T1b (14.1\%), T2b (12.7\%), T3b (4.2\%), T1bN0M1 y T2bN0M1 (2.8\%) у T3c y T1bN1M0 (1.4\%). Cuadro 3 
Cuadro 1. Complicaciones posoperatorias.

\begin{tabular}{|c|c|c|c|c|}
\hline Complicaciones & Frecuencia & Porcentaje & Porcentaje válido & Porcentaje acumulado \\
\hline Hemorragia-hipotensión & 18 & 40 & 40 & 40 \\
\hline Insuficiencia cardiaca & 3 & 6.7 & 6.7 & 46.7 \\
\hline Insuficiencia respiratoria & 7 & 15.6 & 15.6 & 62.2 \\
\hline Neumonía & 3 & 6.7 & 6.7 & 68.9 \\
\hline Neumotórax & 4 & 8.9 & 8.9 & 77.8 \\
\hline Dehiscencia de la herida & 1 & 2.2 & 2.2 & 80 \\
\hline Insuficiencia renal aguda & 5 & 11.1 & 11.1 & 91.1 \\
\hline Atelectasia & 1 & 2.2 & 2.2 & 93.3 \\
\hline Retención aguda de orina & 1 & 2.2 & 2.2 & 95.6 \\
\hline Hematoma & 1 & 2.2 & 2.2 & 97.8 \\
\hline Infección de la herida & 1 & 2.2 & 2.2 & 100 \\
\hline Total & 45 & 100 & 100 & \\
\hline
\end{tabular}

Cuadro 2. Reporte histopatológico.

\begin{tabular}{|c|c|c|c|c|}
\hline Histopatología & Frecuencia & Porcentaje & Porcentaje válido & Porcentaje acumulado \\
\hline Células claras & 62 & 87.3 & 87.3 & 87.3 \\
\hline Células cromófobas & 3 & 4.2 & 4.2 & 91.5 \\
\hline Conductos colectores & 6 & 8.5 & 8.5 & 100 \\
\hline Total & 71 & 100 & 100 & \\
\hline
\end{tabular}

Cuadro 3. Distribución por estadio según la clasificación TNM.

\begin{tabular}{|c|c|c|c|c|}
\hline Estadio & Frecuencia & Porcentaje & Porcentaje válido & Porcentaje acumulado \\
\hline T1aNOMO & 15 & 21.1 & 21.1 & 21.1 \\
\hline T1bN0M0 & 10 & 14.1 & 14.1 & 35.2 \\
\hline T2aNOMO & 15 & 21.1 & 21.1 & 56.3 \\
\hline T2bNOMO & 9 & 12.7 & 12.7 & 69 \\
\hline T3aNOMO & 13 & 18.3 & 18.3 & 87.3 \\
\hline T3bN0M0 & 3 & 4.2 & 4.2 & 91.5 \\
\hline T3cNOMO & 1 & 1.4 & 1.4 & 93 \\
\hline T1bN1M0 & 1 & 1.4 & 1.4 & 94.4 \\
\hline T1bN0M1 & 2 & 2.8 & 2.8 & 97.2 \\
\hline T2bN0M1 & 2 & 2.8 & 2.8 & 100 \\
\hline Total & 71 & 100 & 100 & \\
\hline
\end{tabular}




\section{DISCUSIÓN}

El carcinoma renal es una alteración frecuente en adultos, representa la séptima causa de cáncer en este grupo de pacientes y suele permanecer asintomático hasta que alcanza cierto grado de evolución, lo que dificulta establecer su tratamiento. Esto implica cirugías con mayor grado de dificultad $y$, por consiguiente, alta tasa de complicaciones trans y posoperatorias.

La relación hombre-mujer reportada en este estudio fue de 1.2:1, coincidiendo con el ligero predominio de hombres ya descrito en la bibliografía. El promedio de edad fue de 65.6 años, como lo publican las Guidelines on Renal Cell Carcinoma de 2014; ${ }^{9}$ sin embargo, con una edad mínima de 34 años, muy parecido a lo reportado en los últimos años.

La nefrectomía radical es una intervención compleja que en nuestro estudio se realizó en un promedio de 153.4 minutos, similar a lo señalado en México y Latinoamérica, donde incluso varía de 75 a 330 minutos. El lado más afectado fue el izquierdo (62\%).

El sangrado transoperatorio fue de 1116.4 cc (límites de 100-6000 cc), diferente a lo evidenciado por Varela y sus colaboradores, quienes señalan 1573 cc. ${ }^{10}$ De acuerdo con nuestros datos, $25.4 \%$ de los pacientes requirió trasfusión versus $55.8 \%$ de lo reportado por Arriaga-Morales y su grupo. ${ }^{11}$

Las complicaciones transoperatorias ocurrieron en $25.4 \%$ de los casos, principalmente el desgarro de los vasos de neoformación o adyacentes $(8.5 \%)$, similar a lo señalado por Varela y su equipo $^{10}(22.6 \%)$, pero diferente al estudio de Ballesteros y su grupo $(9.7 \%){ }^{12}$

En relación con las complicaciones posoperatorias, Arriaga y sus colaboradores ${ }^{11}$ encontraron
$42.1 \%$, cifra mayor a la registrada en este estudio $(33.8 \%)$. La hemorragia e hipotensión fueron las complicaciones más frecuentes $(40 \%)$, mientras que la dehiscencia e infección de la herida, sepsis abdominal, atelectasia, retención aguda de orina y hematoma reportaron $2.2 \%$, respectivamente, incluso se manifestaron solas o en conjunto en varios pacientes.

La mayor parte de las complicaciones trans y posoperatorias fueron los grados I, II y III. Del total de pacientes, $11.3 \%$ ameritó ingresar a la unidad de cuidados intensivos. La mortalidad registrada en este estudio fue de $4.3 \%$, cifra más alta que la de Ballesteros y sus coautores (0.8\%).

El estadio tumoral representa el indicador pronóstico más importante. Los principales estadios detectados fueron $\mathrm{T} 1 \mathrm{a}$ y $\mathrm{T} 2 \mathrm{a}(21.1 \%$, respectivamente), lo que coincide con el estudio de Arriaga y su grupo ( $24.6 \%$ para T1 y $28.9 \%$ para T2). El tipo histológico más frecuente fue el carcinoma de células claras (87.3\%), similar a lo reportado en la mayor parte de los estudios.

\section{CONCLUSIÓN}

El carcinoma renal es una alteración que en estadios avanzados origina gran morbilidad y mortalidad, lo que depende de factores intrínsecos del propio paciente y extrínsecos representados por el cirujano y los centros hospitalarios. Entre los primeros deben considerarse: edad, origen de la alteración renal y comorbilidades. Este estudio reportó 25.4 y $33.8 \%$ de complicaciones transoperatorias y posoperatorias, respectivamente, y solo $4.2 \%$ (3 pacientes) de mortalidad en el posoperatorio. La mayor parte de las complicaciones fueron de grado bajo (Gl a GIII). La implementación de un sistema estandarizado para clasificar las complicaciones y asignar un grado de severidad representa una herramienta útil para evaluar los procedimientos quirúrgicos. La nefrectomía 
radical sigue siendo el tratamiento de elección para el carcinoma renal.

\section{REFERENCIAS}

1. Aldaco SF, Pérez PP, Cervantes SG, y col. Mortalidad por cáncer en México 2000-2010: el recuento de los daños. Gaceta Mex Onc 2012;11(6):371-379.

2. Cohen HT, McGovern F. Renal cell carcinoma. N Engl J Med 2005;353(24):77-90.

3. Belldgrun A. Renal cell carcinoma: prognostic factors and patient selection. Eur Urol 2007(Suppl);6:477-483.

4. Belldegrun A. Current treatment in advanced renal cell carcinoma (RCC): Impact of targeted therapies in the management of RCC. Eur Urol supplements 2007;6:484-491.

5. Wein AJ. Campbell Walsh Urology. $10^{\text {th }}$ Ed. Steven C, Campbell and Brian R Lane, editors. Philadelphia: Elsevier/ Saunders, 2012;1413-74.

6. Roigas J. The role of multitargeted therapies in the adjuvant setting in renal cell carcinoma. Eur Urol Suppl 2008;7:63-70.
7. Thomas A, Rini B, Lane B, et al. Response of the primary tumor to neoadjuvant sunitinib in patients with advanced renal cell carcinoma. J Urol 2009;181:518-523.

8. Robson C, Churchill B, Anderson W. The results of radical nephrectomy for renal cell carcinoma. J Urol 1969;101:297.

9. Ljungberg B, Bensalah K, Bex A, et al. Guidelines on renal cell carcinoma. European Association of Urology 2014. [en línea]. Dirección URL: < https://uroweb.org/wp-content/ uploads/EAU-Guidelines-Renal-Cell-Carcinoma-2016. pdf>.

10. Varela R, Donoso DW, Corredor AH. Experiencia de nefrectomía radical en el Instituto Nacional de Cancerología. Bogotá, Colombia. Serie de casos. Urol. Colomb 2010;29(2): 39-44.

11. Arriaga-Morales H, Gutiérrez-Godínez F. Complicaciones de las cirugías renales por cáncer en el Hospital General de México. Rev Mex Urol 2005; 65(3):157-165.

12. Ballesteros-Sampol JJ. Indicaciones y mortalidad de la nefrectomía abierta. Análisis de 681 casos y revisión de la literatura. Arch Esp Urol 2006;59(1):59-70.

\section{AVISO PARA LOS AUTORES}

Revista Mexicana de Urología tiene una nueva plataforma de gestión para envío de artículos: https://www.revisionporpares.com/index.php/RMUrol ahí podrá inscribirse a la base de datos administrada por el sistema Open Journal System (OJS) que ofrece las siguientes ventajas para los autores:

- Subir sus artículos directamente al sistema.

- Conocer, en cualquier momento, el estado de los artículos enviados, es decir, si ya fueron asignados a un revisor, aceptados con o sin cambios, o rechazados.

- Participar en el proceso editorial corrigiendo y modificando sus artículos hasta su aceptación final. 